\title{
ON THE CONVEXITY OF THE LEVEL CURVES OF A POLYNOMIAL
}

\author{
A. W. GOODMAN ${ }^{1}$
}

1. Introduction. Let $z_{1}, z_{2}, \cdots, z_{m}$ be $m$ distinct points in the complex plane and let

$$
P(z)=\prod_{\alpha=1}^{m}\left(z-z_{\alpha}\right)^{k_{\alpha}},
$$

where the exponent $k_{\alpha}$ is a positive integer which gives the multiplicity of the root $z_{\alpha}(\alpha=1,2, \cdots, m)$.

Let $E(c)$ be the open set defined as the set of $z$ for which $|P(z)|<c$, and let $\Gamma(c)$ denote the boundary of $E(c)$. This set $\Gamma(c)$, which is the union of one or several simple closed curves, is just the lemniscate $|P(z)|=c$.

The sets $E(c)$ and $\Gamma(c)$ have been the subject of many papers. In particular the recent paper by Erdös, Herzog, and Piranian [1] raises many interesting questions related to these sets, some of which were answered by Pommerenke [2], [3].

A particular question raised by $\mathrm{H}$. Grunsky and reported in [1] as problem 16 is this: Suppose that the set $E(c)$ has $m$ components, where $m$ is the number of distinct roots of $P(z)$; is it true that each of the $m$ components is a convex set?

It turns out that the answer is no, and we give our counterexample in §2. After this work was completed, Professor Piranian directed me to the paper by Pommerenke [3] who also found a counterexample. But the polynomial proposed by Pommerenke has a rather high degree, and further has a multiple root of high order. This leaves open the question of the convexity of the components of $E(c)$ when all of the roots of $P(z)$ are simple roots and $E(c)$ has $n$ components, where $n$ is the degree of $P(z)$. It turns out that even under these more stringent conditions, the components of $E(c)$ need not be convex, and we give our counterexample in $\$ 3$. Of course the polynomial presented in $\$ 3$, supersedes the one presented in $\$ 2$. However there are some reasons for presenting both polynomials. The first one serves to motivate the construction of the second one, and at the same time it indicates the mechanism, and the difficulties involved in finding a

Presented to the Society, January 24, 1966; received by the editors August 12, 1965.

1 This work was supported by the National Science Foundation, Research Grant GP-4025. 
suitable polynomial with only simple roots. Both polynomials are of fourth degree, and thus represent a gain in economy and simplicity over the Pommerenke counterexample.

2. The first counterexample. Let $P(z)$ be the polynomial

$$
P(z) \equiv\left(z^{2}+1\right)(z-2)^{2} .
$$

Then $P^{\prime}(z)=2(z-2)\left(2 z^{2}-2 z+1\right)$ and hence $P^{\prime}(z)$ vanishes for $z_{1}^{*}, z_{2}^{*}=(1 \pm i) / 2$ and $z_{3}^{*}=2$. If we set $c=\left|P\left(z_{1}^{*}\right)\right|$, we see that by symmetry $c=\left|P\left(z_{2}^{*}\right)\right|$, and for this $c$, the curve $\Gamma(c)$ has two double points, one at $z_{1}^{*}$ and the other at $z_{2}{ }^{*}$. Consequently $E(c)$ has three components as required by the conditions of our problem. Let $E_{3}(c)$ be that component of $E(c)$ that contains the point $z_{3}=2$. Both the points $z_{1}^{*}$ and $z_{2}^{*}$ are on the boundary of $E_{3}(c)$, so that if $E_{3}(c)$ were convex, its closure would contain the line segment joining $z_{1}{ }^{*}$ and $z_{2}{ }^{*}$ and in particular would contain the point $x^{*}=R\left(z_{1}^{*}\right)=1 / 2$. Now direct computation gives

$$
c=\left|P\left(z_{1}^{*}\right)\right|=\left|\left(\frac{i}{2}+1\right)\left(\frac{i}{2}-\frac{3}{2}\right)^{2}\right|=\frac{5 \sqrt{ } 5}{4},
$$

and

$$
\left|P\left(x^{*}\right)\right|=\left(\frac{1}{4}+1\right)\left(\frac{1}{2}-2\right)^{2}=\frac{45}{16} .
$$

Since $45 / 16>5 \sqrt{ } 5 / 4$, it follows that $x^{*}$ lies outside the closure of $E_{3}(c)$ and hence $E_{3}(c)$ is not convex. A rough sketch of $\Gamma(c)$ will show why $E_{3}(c)$ is not convex.

3. The second counterexample. The polynomial $P(z)$ defined in $\$ 2$ has a double root at $z=2$. Conceptually, it is an easy matter to split the double root into two simple roots and still achieve a nonconvex component. However the direct computation is involved. Whereas it was a simple matter to locate the roots of $P^{\prime}(z)$ in our first counter example, this is not true when the 4 roots are distinct. Hence we start with $Q^{\prime}(z)$ with known roots, and in fact we never need to find the roots of $Q(z)$ explicitly.

Set

$$
Q^{\prime}(z)=12\left(z^{2}+1\right)(z-a), \quad a>0 .
$$

Then

$$
Q(z)=3 z^{4}-4 a z^{3}+6 z^{2}-12 a z+b,
$$

where $b>0$ is a constant to be determined. Under these conditions, 
$Q(z)$ has no roots that are real and negative. But the critical points are at $z_{1}^{*}, z_{2}{ }^{*}= \pm i$ and $z_{3}^{*}=a$. Hence by the Gauss-Lucas Theorem (the roots of $Q^{\prime}$ lie in the convex hull of the roots of $Q$ ) it is clear that two of the roots of $Q(z)$ are complex conjugates, $z_{1}$ and $z_{2}$, with $R\left(z_{1}\right)=R\left(z_{2}\right)<0$. Since $Q(0)=b>0$ and $Q(z) \rightarrow+\infty$ as $z \rightarrow+\infty$, we can locate the other two roots $z_{3}$ and $z_{4}$ if we know that $Q(a)<0$. Consequently one necessary condition on the constants $a$ and $b$ is that

$$
Q(a) \equiv 3 a^{4}-4 a^{4}+6 a^{2}-12 a^{2}+b<0
$$

or

$$
0<b<a^{2}\left(a^{2}+6\right) .
$$

When the inequality ( 7$)$ is satisfied the polynomial $Q(z)$ has 4 distinct roots, $z_{1}, z_{2}, z_{3}$, and $z_{4}$, with $0<z_{3}<a<z_{4}$.

Just as for the polynomial of $\S 2$, we select $c$ to be $\left|Q\left(z_{1}^{*}\right)\right|$ where $z_{1}^{*}=i$, one of the critical points of $Q(z)$. An easy computation gives

$$
c^{2}=|Q(i)|^{2}=(b-3)^{2}+64 a^{2} .
$$

With this selection of $c$, the set $E(c)$ has at least three components (and possibly four). Two of the components $E_{1}$ and $E_{2}$ are determined by the roots $z_{1}$ and $z_{2}$.

Since $Q(z)$ has real coefficients, we can appeal to the symmetry of $E(c)$ about the real axis. It follows from this symmetry that the remainder set $E(c)-E_{1} \cup E_{2}$, will consist of two components if and only if $|Q(a)| \geqq c$. This occurs if and only if

$$
\left(a^{2}\left(a^{2}+6\right)-b\right)^{2} \geqq(b-3)^{2}+64 a^{2} .
$$

For computation, it is convenient to write this inequality as

$$
\left(a^{4}+6 a^{2}-3\right)\left(a^{4}+6 a^{2}-2 b+3\right)>64 a^{2} .
$$

Assuming that (8) is satisfied, $E(c)$ will have four components $E_{1}, E_{2}$, $E_{3}$ and $E_{4}$ where each $E_{\alpha}$ contains $z_{\alpha}, \alpha=1,2,3,4$.

Following the pattern of proof used in $\$ 2$, the component $E_{3}$ will not be convex if $|Q(0)|>c \equiv|Q(i)|$. This is the case if and only if

$$
b^{2}>(b-3)^{2}+64 a^{2}
$$

or

$$
6 b>9+64 a^{2} .
$$

It only remains to select the positive constants $a$ and $b$ so that the inequalities (7), (8), and (9) are satisfied. This is the case if $a=5$ and $b=303$. Very likely 5 is the smallest integer that can be used for $a$, while $b=303$ is probably too large. Using these constants in equation 
(6) we have the

TheOREM. Let $c^{2}=91,600$ and let

$$
Q(z)=3 z^{4}-20 z^{3}+6 z^{2}-60 z+303 .
$$

Then the polynomial $Q(z)$ has four distinct roots and one of the components of $E(c)$ is not convex.

To obtain the normalization of equation (1) we merely divide $Q(z)$ and $c$ by 3 .

4. Some open questions. One question that readily occurs is to determine the maximum number of nonconvex components of $E(c)$ as a function of $n$, the degree of the polynomial. One can also search for some additional restriction on the polynomial that will insure that each component of $E(c)$ is convex. One can also impose some type of measure of the nonconvexity of a set, and try to determine some maximum for this measure. Certainly in the two examples of this paper the components $E_{3}(c)$ do not miss being convex by very much.

Suppose again that for the polynomial defined by equation (1), the set $E(c)$ has $m$ components. It seems to me that each component $E_{\alpha}$ must be starlike with respect to the root $z_{\alpha}$ which it contains. However, I have not been able to prove this conjecture.

The referee of this paper suggested that the polynomial $P(z)$ $=z\left(z^{5}-1\right)$ also furnishes an interesting counterexample. When $c=5 / 6^{6 / 5}$ the curve $\Gamma(c)$ contains 5 double points where the curve intersects itself at right angles. Since these points all lie on the boundary of the component of $E(c)$ that contains $z=0$, that component cannot be convex.

The referee does not share the author's feeling that when the number of components equals the number of distinct roots, then the components must be starlike. Rather he feels that one of the components can approximate any shape. If this latter turns out to be the case, it would be interesting to know the smallest degree for which starlikeness of each component no longer holds.

\section{REFERENCES}

1. P. Erdös, F. Herzog and G. Piranian, Metric properties of polynomials, J. Analyse Math. 6 (1958), 125-148.

2. C. Pommerenke, On some problems by Erdös, Herzog, and Piranian, Michigan Math. J. 6 (1959), 221-225.

3. - On metric properties on complex polynomials, Michigan Math. J. 8 (1961), 97-115.

The University of South Florida, Tampa 